\title{
Development of an optimal operation approach in MPC framework for heavy haul trains
}

\author{
Lijun Zhang and Xiangtao Zhuan
}

\begin{abstract}
An operation control approach for heavy haul trains to optimize their performance, including operation safety, service quality and energy consumption, is proposed. Following a model predictive control method, the controller is capable of scheduling a train to operate optimally during a long section of the rail track. In the cost function, two penalty factors are presented, one for the braking forces and one for coupler damping effects. The penalty for braking forces is employed to reduce energy waste incurred by braking. The penalty for coupler damping is introduced to alleviate the cyclic vibration of couplers, which link adjacent cars in the train. The damping penalty is also expected to reduce energy wasted by coupler damping and corresponding maintenance/replacement cost of the dampers. In addition, the weight of the velocity tracking term in the objective function is modified to vary dynamically according to the train's velocity to improve the train's overall performance. Simulations verify the effectiveness of the proposed control approach. Discussions over the impacts of the two penalty factors and dynamic weight method are provided together with some suggestions on their applications.
\end{abstract}

Index Terms-Operation control, heavy haul trains, model predicative control, penalty factors, dynamic weighting.

\section{INTRODUCTION}

Operating railway systems in a safe and energy-efficient way has drawn a lot of attention since the beginning of the railway industry. In particular, demand for energy saving and environmental conservation has brought about a new upsurge of researches addressing the energy consumption of railway vehicles. Efforts have been made in roadside facility improvement [1], [2], train scheduling under complex networks [3], [4], [5], train operation control [6], [7], etc. The focus of this paper is to develop an optimal train operation strategy which optimizes the train's performance while reducing its operational costs (energy cost and maintenance/repair cost). The train performance indicators used in this study include operation safety determined by in-train forces, service quality in terms of punctuality characterized by velocity tracking [8], coupler fatigue characterized by its cyclic vibration, and energy consumption.

This investigation focuses on heavy haul train, capable of electronically controlled pneumatic braking and independent distributed power (ECP/iDP) operation mode, similar to the one described by [9]. In the scope of control of trains, many existing approaches formulate such a control problem into

L. Zhang was with the School of Power and Mechanical Engineering, Wuhan University, Wuhan 430072, China. He has been with the Department of Electrical, Electronic and Computer Engineering of University of Pretoria since August 2012. X. Zhuan is with the School of Power and Mechanical Engineering, Wuhan University, Wuhan 430072, China. Email: lijun.zhang@up.ac.za, xtzhuan@whu.edu.cn. various optimization problems with different concerns. Most of them minimize the train's energy usage during its travel, regardless of the control approach employed [10].

In 1982, the in-train dynamics of trains were studied [11]. Closed-loop control method is employed to optimize the interplay between in-train forces and speed tracking in order to optimize the speed tracking and energy consumption [12]. In 2006, a closed-loop controller was proposed, which considered the train's operation safety in terms of in-train forces and service quality in terms of velocity tracking into account [13].

Before 2006, in-train dynamics were usually ignored in controller design. There are two reasons why many researchers ignored in-train dynamics of a train, which is actually a very important aspect of a train's operation safety. The first reason is that the train lengths were much shorter in the past years, when locomotives in the train can apply sufficient power to pull/push the train to keep the in-train forces in safe range. The second reason is that since energy consumption was the main concern, most of those researchers modeled the trains as a single mass point because in-train dynamics are not directly related to energy consumption [7]:

$$
\left\{\begin{array}{l}
\dot{x}(t)=v(t) \\
\dot{v}(t)=f(u(t), v(t))-r(x(t), v(t)),
\end{array}\right.
$$

where $x(t)$ and $v(t)$ are the position and velocity of the train at time $t, u(t)$ is the control output (power or force), $f(u(t), v(t))$ is the acceleration/deceleration with respect to $u(t)$, and $r(x(t), v(t))$ is the deceleration with respect to the resistance. It is clear that the velocity and position are dependent on time, however, this dependence is omitted in the remaining parts of this paper for the sake of simplicity.

Control methods using model (1) tend to optimize a train's performance by optimizing the switching time between different operation modes, (such as power, coast and brake), without considering in-train dynamics [7], [10], [14], [15], [16], [17].

Based on the single mass model (1), a cost function in the optimization problem is usually defined as

$$
J=\int_{0}^{T} p d t
$$

where $p$ is the power of the train, and $[0, T]$ denotes the travel time interval. From this cost function, it can be seen that only energy consumption is taken into account in the optimization. The existence of an optimal operation strategy of a train is proved by [18] also with the single mass point model. In the later study of $\mathrm{Li}$ et al. [19], carbon emission has been taken into account, to optimize the train's performance in view of the 
carbon emission trading scheme formulated by the European Union; but no in-train dynamics were considered.

Heavy haul trains in operation today normally consist of more than 200 cars, and in-train forces are the direct cause of coupler damages which may lead to train operation disasters like derailment. Thus the in-train forces should be considered and manipulated with care in order to reduce a train's maintenance cost, and most importantly to ensure operation safety. It was verified in Zhuan's Ph.D thesis [20] that using a single mass point model in a long train leads to unreasonable power distribution throughout the train and results in unacceptable in-train forces. It is concluded in [20] that the single mass model results in large in-train forces when the train is running over the top of a hill where the front part of train is running downhill while the rear part is running uphill. In view of this, the so called cascade mass point model was employed by [21], which models the whole train as individual masses (cars) interconnected by couplers

$$
\left\{\begin{array}{l}
m_{i} \dot{v}_{i}=u_{i}+f_{i n_{i-1}}-f_{i n_{i}}-f_{a_{i}}, \quad i=1, \ldots, n, \\
x_{i n}=v_{i}-v_{i+1}, \quad i=1, \ldots, n-1,
\end{array}\right.
$$

where $n$ is the number of cars in the train, $m_{i}$ is the $i$-th car's mass and, $v_{i}$ and $u_{i}$ are the speed and effort of the $i$ th car. The variable $f_{a_{i}}=f_{\text {aero }_{i}}+f_{p_{i}}$, in which $f_{\text {aero }}$ in $=$ $m_{i}\left(c_{0_{i}}+c_{1_{i}} v_{i}+c_{2_{i}} v_{i}^{2}\right)$ is the $i$-th car's rolling resistance and aerodynamic force, and $f_{p_{i}}=f_{g_{i}}+f_{c_{i}}$ is the force due to the track slope and curvature on which the $i$-th car is running. The variable $f_{i n_{i}}=k_{i} x_{i n_{i}}+d_{i} \dot{x}_{i n_{i}}$ is the in-train force between the $i$-th and $i+1$-th cars, in which $x_{i n}$ is the relative displacement of the $i$-th and $i+1$-th cars and $k_{i}$ and $d_{i}$ are the elastic and damping coefficients of coupler $i$.

Taking advantage of model (2), heavy haul trains' operation controllers minimizing in-train forces, velocity tracking errors, and energy consumptions were studied [13], [9], [22]. The highlight of these studies is that they not only address the energy consumption aspect of heavy haul trains, but other safety and service quality related aspects are also considered. The cost function defined in those studies are similar to the following one:

$$
J=\int_{t_{0}}^{t_{f}}\left(\sum_{i=1}^{n-1} K_{f} f_{i n_{i}}^{2}+\sum_{i=1}^{n} K_{e} u_{i}^{2}+\sum_{i=1}^{n} K_{v}\left(v_{i}-v_{r}\right)^{2}\right) d t
$$

where $K_{f}, K_{e}$ and $K_{v}$ are weights and $v_{r}$ is reference speed, $\left[t_{0}, t_{f}\right]$ denotes the optimization interval.

Closed-loop controllers proposed in those papers have made full use of the train's capabilities to improve performance. For instance, ECP/iDP operation strategy, which is verified to give the best performance compared to other train brake/traction strategies, is adopted so that manipulation of the train can be most effective [13].

However, it is noticed that all those studies schedule a train according to its current running condition including velocity, position and track information. It is desirable to optimize the train's operation during a long travel rather than at a specific position such that the future states of the train and the incoming track information are accounted for. In view of this, model predictive control (MPC) approaches were introduced in [23] and [24] to optimize the trains behavior over a chosen period taking into account all physical and operational constraints. The MPC approach, with its intrinsic advantages of tackling constrained operation control problem and optimizing system behavior by looking ahead, has been successfully applied to many industrial systems [25], [26], [27], [28]. The work presented in [23] and [24] show the resultant improvement in train performance in terms of velocity tracking, in-train force minimization and energy consumption reduction.

Further, there are three factors not considered in previous works including [7], [9], [23] and [24]. Firstly, it is concluded in [29] that statistically, $10-20 \%$ of the energy consumed by a train is used to compensate kinetic energy loss resulted from braking. This implies that during a train's operation, the control strategy must take energy dissipated by braking into consideration. Secondly, cyclic vibration of couplers, as the main cause of coupler fatigue [30], was not addressed in [7], [9], [23]. Only the maximum absolute value of intrain forces were accounted for. Thirdly, the weight factors for different train performance indicators are all fixed values, whereas it is necessary to change the factor $K_{v}$, corresponding to the velocity tracking status. This is because if $K_{v}$ is fixed, the optimization tends to direct the train to stop under some circumstances (see details in Section II-B).

To tackle the above-mentioned issues, two penalty factors $K_{b}$ and $K_{d}$ are presented in this paper to balance braking forces and coupler damping effects of a train. Also, a dynamically varying weight factor is applied to the velocity tracking indicator. The advantages of those three modifications, including reduced energy consumption, reduced maximum in-train force and reduced coupler cyclic vibration of the train, are verified by simulations.

Although the speed profile of a train on a given track should and can be optimized as done in [31] and [32], it is out of the scope of this paper and is not discussed. Only speed tracking with respect to a given reference speed profile is investigated.

The remainder of this paper is organized as follows: train optimal control problem is presented in Section II and an MPC approach with penalty factors, and dynamically varying $K_{v}$ is given in Section III. Simulation results are shown in Section IV to demonstrate the effectiveness of the proposed approach, while hints on the application of the presented penalty factors and dynamic $K_{v}$ are provided in Section V. Section VI concludes this paper.

\section{PROBLEM}

The optimal control problem of heavy haul trains is discussed in this section. Firstly, a general problem formulation is given with the objective to minimize the train's energy consumption while the operation safety and service quality are ensured. Then the cost function of the problem is modified to take some other key aspects of the train operation into consideration.

\section{A. Problem in general}

The train model and operation constraints are the same as detailed in the previous works [23], [24], [33]. The cascade 
mass point model (2) and operation constraints described by (4) are used.

$$
\begin{gathered}
u_{i}^{l} \leq u_{i} \leq u_{i}^{u}, \quad i=1,2, \ldots, n \\
\Delta u_{l_{j}}^{l} \leq \Delta u_{l_{j}} \leq \Delta u_{l_{j}}^{u}, \quad j=1,2, \ldots, m \\
f_{k}^{l} \leq f_{i n_{k}} \leq f_{k}^{u}, \quad k=1,2, \ldots, n-1
\end{gathered}
$$

where $u_{i}^{l}, u_{i}^{u}$ are lower and upper limitations of the $i$ th car's effort (control variable), the variables $\Delta u_{l_{j}}^{l}$ and $\Delta u_{l_{j}}^{u}$ are the lower and upper bounds of the $j$ th locomotive's power change within one sampling period. The variable $l_{j}, j=1,2, \ldots, m$ denotes the $j$ th locomotive's position in the train, and $f_{k}^{l}, f_{k}^{u}$ are the lower and upper bounds for the $k$ th in-train force.

The train model is further transferred by denoting a new control variable $u_{i}^{\prime}=u_{i}-\left(m_{i} c_{0_{i}}+m_{i} c_{2_{i}} v_{i}^{2}+f_{p_{i}}\right)$ that make the train dynamics to take origin as an equilibrium point. Then with damping effect ignored (let $d_{i}=0$ ), the model is linearized to be as follows

$$
\left\{\begin{array}{l}
m_{i} \dot{v}_{i}=u_{i}^{\prime}+k_{i-1} x_{i-1}-k_{i} x_{i}-m_{i} c_{1_{i}} v_{i}, i=1, \ldots, n, \\
\dot{x}_{i n_{i}}=v_{i}-v_{i+1}, i=1, \ldots, n-1
\end{array}\right.
$$

Choosing $x=\left[v_{1}, v_{2}, \cdots, v_{n}, x_{i n_{1}}, x_{i n_{2}}, \cdots, x_{i n_{n-1}}\right]^{T}$ as state variable, and $u^{\prime}=\left[u_{1}^{\prime}, u_{2}^{\prime}, \ldots, u_{n}^{\prime}\right]^{T}$ as decision vector (control variable), the transferred dynamical equation becomes linear and can be written as follows

$$
\dot{x}=A_{p} x+B_{p} u^{\prime},
$$

where $A_{p}=\left[\begin{array}{ll}A_{11} & A_{12} \\ A_{21} & A_{22}\end{array}\right], B_{p}=\left[\begin{array}{c}B_{11} \\ 0_{n \times n}\end{array}\right]$. To be exact, $A_{11}=-\operatorname{diag}\left\{c_{1_{1}}, c_{1_{2}}, \cdots, c_{1_{n}}\right\}, A_{22}=0_{n \times n}, B_{11}=$ $\operatorname{diag}\left\{\frac{1}{m_{1}}, \frac{1}{m_{2}}, \cdots, \frac{1}{m_{n}}\right\}$,

$$
\begin{aligned}
& A_{12}= {\left[\begin{array}{cccc}
-\frac{k_{1}}{m_{1}} & 0 & \cdots & 0 \\
\frac{k_{1}}{m_{2}} & -\frac{k_{2}}{m_{2}} & \cdots & 0 \\
\vdots & \vdots & \ddots & \vdots \\
0 & 0 & \cdots & \frac{k_{n-1}}{m_{n}}
\end{array}\right], \text { and } } \\
& A_{21}=\left[\begin{array}{cccccc}
1 & -1 & 0 & \cdots & 0 & 0 \\
0 & 1 & -1 & \cdots & 0 & 0 \\
\vdots & \vdots & \vdots & \ddots & \vdots & \vdots \\
0 & 0 & 0 & \cdots & 1 & -1
\end{array}\right]
\end{aligned}
$$

Then the above continuous time domain state-space equation can be discretized by the zero-order hold method with sampling period $T_{s}$ to have the following form

$$
x(k+1)=A x(k)+B u^{\prime}(k),
$$

where $x(k)$ consists of all car's current velocities and the relative displacements of neighbouring cars in a train, $x(k)=$ $\left[v_{1}(k), \cdots, v_{n}(k), x_{i n_{1}}(k), \cdots, x_{i n_{n-1}}(k)\right]^{T}$ and $u^{\prime}(k)=$ $\left[u_{1}^{\prime}(k), u_{2}^{\prime}(k), \ldots, u_{n}^{\prime}(k)\right]^{T}$ are the decision vector. The system matrices are: $A=e^{A_{p} T_{s}}$ and $B=\int_{0}^{T_{s}} e^{A_{p} \tau} d \tau B_{p}$.

With the state space train model (5), with constants and higher order terms eliminated, the cost function (3) can be transformed into its approximated form as follows

$$
J=\sum_{k=1}^{N_{p}} x^{T} Q_{x} x+u^{\prime T} R u^{\prime}+F_{1}^{T} x+F_{2}^{T} u^{\prime},
$$

where $N_{p}$ is the optimization horizon,

$$
\begin{gathered}
Q_{x}(i, i)=\left\{\begin{array}{l}
K_{v}, i=1, \ldots, n, \\
K_{f} k_{i-n}^{2}, i=n+1, \ldots, 2 n-1,
\end{array}\right. \\
F_{1}^{T}=-2 K_{v} v_{r}[\overbrace{1, \ldots, 1}^{n}, \overbrace{0, \ldots, 0}^{n-1}, F_{2}^{T}=2 K_{e}\left[m_{1} c_{0_{1}}+\right. \\
\left.f_{p_{1}}, \ldots, m_{n} c_{0_{n}}+f_{p_{n}}\right], \text { and } R=K_{e} I_{n \times n} .
\end{gathered}
$$

In summary, the optimal heavy haul train operation problem is to minimize cost function (6) subject to train dynamics (5) and operational constraints (4).

\section{B. Updates of the cost function}

The cost function of the train operation problem suggests that it is a weighted sum optimization problem. So changing the weights can result in a different performance. In addition, there are some other factors that have impacts on the optimized performance but are not included in (3) [34]. In accordance with the scope of this paper stated in Section I, the following updates are applied to the cost function (3).

1) A penalty factor $K_{b}$ is introduced to penalize the wagons' braking efforts because as stated in [29], statistically $10-20 \%$ of the energy consumed by a train is used to compensate kinetic energy loss resulted from braking.

2) A factor $K_{d}$ is adopted to penalize the cyclic vibration of couplers in order to alleviate coupler fatigue as well as to bring down energy consumed during coupler damping.

3) A dynamically varying $K_{v}$ with respect to the train's current speed tracking state is used instead of a fixed one as in [22], [23] to improve the train's performance. The velocity tracking related term in (3) is $\sum_{i=1}^{n} K_{v}\left(v_{i}-\right.$ $\left.v_{r}\right)^{2}$. While $v_{r}$ is a constant, the minimization of $J$ tries to minimize $v_{i}$ when $\sum_{i=1}^{n} K_{v}\left(v_{i}-v_{r}\right)^{2}$ is relatively larger compared to the other parts of (3). This causes the optimization to bring the train to a standstill $\left(v_{i}=0\right)$. In such a way, the train's performance regarding velocity tracking and other aspects would all deteriorate. Therefore, it is desirable to change $K_{v}$ dynamically according to the train's speed tracking status, in order to improve the train's overall performance.

From this point onward in this section, the focus will be on mathematically representing the three updates of the objective function given above.

1) Braking penalization: Introducing a braking penalty $K_{b}$ is straightforward. Applying the following updates to the energy related term in the cost function:

$$
\sum_{i=1}^{n} K_{e} u_{i}^{2} \rightarrow K_{e}\left(\sum_{j=1}^{m} u_{l_{j}}^{2}+K_{b} \sum_{i=1, i \neq l_{j}}^{n} u_{i}^{2}\right),
$$

will add a braking penalty factor $K_{b}$ for wagons in the train. It can be seen that if $K_{b}=1$, this modification is removed and no extra penalty for braking is presented. The reason why the penalty factor does not apply to locomotives is that locomotives can be capable of regenerative braking which can use the energy generated during braking in the next acceleration and is energy-efficient already. 
2) Damping penalization: As for $K_{d}$, a new term is added in the cost function:

$$
\begin{aligned}
K_{d} \sum_{i=1}^{n-1} \dot{x}_{i n_{i}}^{2} & =K_{d} \sum_{i=1}^{n-1}\left(v_{i}-v_{i+1}\right)^{2} \\
& =K_{d} \sum_{i=1}^{n-1}\left(v_{i}^{2}+v_{i+1}^{2}\right)-2 K_{d} \sum_{i=1}^{n-1} v_{i} v_{i+1} \\
& =x^{T} Q_{d} x,
\end{aligned}
$$

where

$$
Q_{d}=\left[\begin{array}{cc}
D_{n \times n} & 0 \\
0 & 0
\end{array}\right]-2 K_{d} P_{1}^{T} P_{2}^{T},
$$

in which $D$ is a $n \times n$ diagonal matrix with elements

$$
D(i, i)=\left\{\begin{array}{l}
2 K_{d}, i=2,3, \ldots, n-1 \\
K_{d}, \text { otherwise }
\end{array}\right.
$$

and

$$
P_{1}=\left[\begin{array}{cc}
0 & I_{2 n-2 \times 2 n-2} \\
1 & 0
\end{array}\right], P_{2}=\left[\begin{array}{cc}
I_{n-1 \times n-1} & 0 \\
0 & 0
\end{array}\right] .
$$

It is noted that if $K_{d}$ is set to 0 , no penalty for the coupler's damping effect is applied.

3) Dynamic velocity tracking: In this subsection, a Gaussian-like function is introduced to adjust $K_{v}$ dynamically according the train's current velocity tracking result, i.e., applying the following change to the $K_{v}$ in the cost function:

$$
K_{v}^{\prime}=K_{v}\left(1-e^{-\frac{\left(v-v_{r}\right)^{2}}{c^{2}}}\right),
$$

in which $c$ determines the rate of change of $K_{v}^{\prime}$.

Ultimately, applying changes introduced in (7), (8), and (9), the cost function (3) is rewritten as follows

$$
\begin{aligned}
J= & \sum_{k=1}^{N_{p}}\left(K_{f} \sum_{i=1}^{n-1} f_{i n_{i}}^{2}+K_{v}^{\prime} \sum_{i=1}^{n}\left(v_{i}-v_{r}\right)^{2}\right. \\
& \left.+K_{e}\left(\sum_{k=1}^{m} u_{l_{k}}^{2}+K_{b} \sum_{i=1, i \neq l_{k}}^{n} u_{i}^{2}\right)+K_{d} \sum_{i=1}^{n-1} \dot{x}_{i n_{i}}^{2}\right) .
\end{aligned}
$$

Similarly, with the discrete-time train model (5), the above cost function is transformed into

$$
J=\sum_{k=1}^{N_{p}} x^{T}\left(Q_{x}+Q_{d}\right) x+u^{\prime T} R u^{\prime}+F_{1}^{T} x+F_{2}^{T} u^{\prime},
$$

where $R$ is a $n \times n$ diagonal matrix in which

$$
\begin{gathered}
R(i, i)=\left\{\begin{array}{l}
K_{e}, i=l_{j}, j=1,2, \ldots, m ; \\
K_{b} K_{e}, \text { otherwise } ;
\end{array}\right. \\
Q_{x}(i, i)=\left\{\begin{array}{l}
K_{v}^{\prime}, i=1, \ldots, n ; \\
K_{f} k_{i-n}^{2}, i=n+1, \ldots, 2 n-1 ;
\end{array}\right.
\end{gathered}
$$

while $F_{1}^{T}$ and $F_{2}^{T}$ are the same as in equation (6).

Consequently, the cost function in the optimization problem of the train operation is changed from the general one (6) to the updated one (10). This problem can be solved by a quadratic programming (QP) method as can be seen that it has a quadratic cost function.

\section{MPC'S APPLICATION TO HEAVY HAUL TRAINS}

An MPC approach for heavy haul trains has been proposed in our previous study [23] in order to optimize the trains' operation during a long journey. The MPC controller manipulates the traction/braking forces for the train by solving an optimization problem that minimizes the chosen indicators over a predicted interval. At each time when a control is required, the controller predicts future states of the train, according to information available, for a chosen interval. After the prediction is done, the optimization problem is solved, which yields a sequence of control signal. The first element in this sequence is then applied to the train and the rest are discarded. When the next control instance arrives, the procedure comprising state prediction, solving of optimization problem, and implementation of the first control element, is repeated. By using a properly chosen optimization horizon, the optimized result is locally optimal and smooths the train's performance over a long travel.

The MPC controller, as stated above, requires prediction of the train's states in an optimization horizon and solving of a properly formulated optimization problem. The scheme of state prediction based on the train's dynamics, and optimization problem formulation in the predicted horizon are presented in the following.

State prediction is done as follows

$$
X=F x(k)+\Phi U,
$$

where $X=\left[x(k+1 \mid k)^{T}, x(k+2 \mid k)^{T}, \ldots, x(k+N p \mid k)^{T}\right]^{T}$ and $U=\left[u^{\prime}(k)^{T}, u^{\prime}(k+1)^{T}, \ldots, u^{\prime}\left(k+N_{c}-1\right)^{T}\right]^{T}$ are predicted state vector and corresponding optimal control signal based on the current state, and

$$
F=\left[\begin{array}{c}
A \\
A^{2} \\
\vdots \\
A^{N_{p}}
\end{array}\right], \Phi=\left[\begin{array}{cccc}
B & 0 & \ldots & 0 \\
A B & B & \ldots & 0 \\
\vdots & \vdots & \ldots & \vdots \\
A^{N_{p}-1} B & A^{N_{p}-2} B & \ldots & A^{N_{p}-N_{c}} B
\end{array}\right] .
$$

To formulate the optimization problem, the objective function and constraints must also be extended to incorporate the predicted train states. With help of (11), the trains' operational constraints in the optimization horizon are formulated. This is detailed in [24], and a brief description is given below.

Regarding the constraints on in-train forces, with the assumption that the relationship between in-train forces, $f_{i n}$, and relative displacement of two adjacent cars, $x_{i n}$, is $f_{i n_{i}}=$ $k_{i} x_{i n_{i}}$, the constraints on in-train forces is actually constraints on the state variables because

$$
x_{i n}(k)=Z x(k),
$$

where $Z=\left[\begin{array}{ll}0_{n-1 \times n} & I_{(n-1) \times(n-1)}\end{array}\right]$.

As for constraints on the amplitude and the variation of the control variable, they are both related to the control variable $u^{\prime}$. It is true that the limits of variation of locomotives' efforts, $\Delta u_{l_{j}}$, can be represented by limits of the amplitude of $u_{l_{j}}$, if $u_{l_{j}}^{u}$ and $u_{l_{j}}^{l}$ were updated to a proper value according to the limit of $\Delta u_{l_{j}}$ before each loop of the optimization problem. In such a way, the constraints on the amplitude and the rate of 
change of $u_{l_{j}}$ can be merged and represented by constraints on the amplitude of $u^{\prime}$ only.

Therefore, using (12) together with (11), the train's operational constraints (4) are finally formulated as

$$
M U \leq \gamma,
$$

where

$$
\begin{gathered}
M=\left[\begin{array}{c}
I_{\left(n N_{c} \times n N_{c}\right)} \\
-I_{\left(n N_{c} \times n N_{c}\right)} \\
\Phi_{x} \\
-\Phi_{x}
\end{array}\right], \gamma=\left[\begin{array}{c}
U_{\left(n N_{c} \times 1\right)}^{U} \\
-U_{\left(n N_{c} \times 1\right)}^{L} \\
X_{(n-1) N_{c} \times 1}^{U}-F_{x} x(k) \\
-X_{(n-1) N_{c} \times 1}^{L}+F_{x} x(k)
\end{array}\right], \\
F_{x}=\left[\begin{array}{c}
A \\
A^{2} \\
\vdots \\
A^{N_{c}}
\end{array}\right], \Phi_{x}=\left[\begin{array}{cccc}
Z B & 0 & \ldots & 0 \\
Z A B & B & \ldots & 0 \\
\vdots & \vdots & \ldots & \vdots \\
Z A^{N_{c}-1} B & Z A^{N_{c}-2} B & \ldots & Z B
\end{array}\right],
\end{gathered}
$$

in which $U^{U}, U^{L}, X^{U}$ and $X^{L}$ are the upper and lower bounds of $u^{\prime}$ and $x_{i n}$ in the optimization horizon, respectively.

Meanwhile, it is seen in equation (6) that $F_{1}$ is related to speed profile, $v_{r}$, and $F_{2}$ is related to $f_{p}$. These two are directly related to the train's position at a given track. Denote the velocity elements in the state variable $x(k+i \mid k)$ as $v(k+i \mid k)$, and the position of the train at time $k$ as $p(k)$, the train's position at time $k+i$ can be approximately calculated as

$p(k+i \mid k)=p(k)+0.5 T_{s} \sum_{j=1}^{i}(v(k+j-1 \mid k)+v(k+j \mid k))$.

Note that $v(k+j \mid k)$ can be obtained according to (11).

Then, the matrix $F_{1}$ and $F_{2}$ can be calculated during the prediction procedure owing to the fact that both $v_{r}$ and $f_{p}$ are only dependent on $p$.

As a result, in the optimization, prediction of $F_{1}$ and $F_{2}$ could be done as follows

$$
\begin{aligned}
\bar{F}_{1} & =\left[F_{1}(k)^{T}, \ldots, F_{1}\left(k+N_{p}\right)\right]^{T}=h(p), \\
\bar{F}_{2} & =\left[F_{2}(k)^{T}, \ldots, F_{2}\left(k+N_{p}\right)\right]^{T}=g(p) .
\end{aligned}
$$

where $F_{1}(k+i)$ and $F_{2}(k+i)$ are the discrete values of $F_{1}$ and $F_{2}$ in equation (6), respectively. The functions $h(\cdot)$ and $g(\cdot)$ stand for the mapping from $p$ to $\bar{F}_{1}$ and to $\bar{F}_{2}$, respectively.

In summary, the train operation optimization problem in the MPC framework is given as follows,

\section{minimize}

$$
J=U^{T} H U+2 U^{T} f
$$

subject to train dynamics (5) and constraints (13), where $H=\Phi^{T} \bar{Q} \Phi+\bar{R}, f=\Phi^{T} \bar{Q} F x(k)+\frac{1}{2}\left(\Phi^{T} \bar{F}_{1}+\bar{F}_{2}\right)$, in which $\bar{Q}=\operatorname{diag}\left[Q_{x}+Q_{d}, \ldots, Q_{x}+Q_{d}\right], \stackrel{2}{R}=\operatorname{diag}[R, \ldots, R]$.

The MPC algorithm for solving this problem works as follows

MPC Algorithm: Input $\left(x(0), u^{l}, u^{u}, \Delta u^{l}, \Delta u^{u}\right.$, $f^{l}, f^{u}, v_{r}, T_{s}$ and track information).

1) Let $k=0$.

2) Feedback $x(k)$, and apply QP method to find the optimal control sequence which contains $N_{c}$ control variables $U_{\text {opt }}=\left\{u(k), u(k+1), \cdots, u\left(k+N_{c}-1\right)\right\}$.

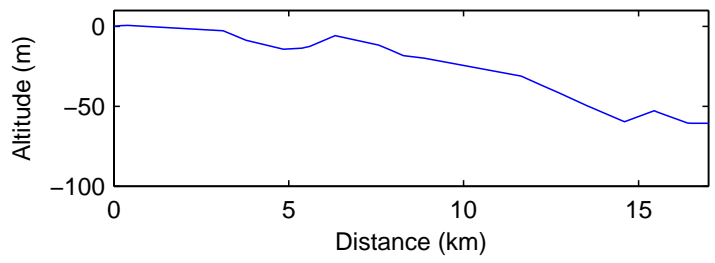

Fig. 1: Track information

3) Implement $u(k)$ at the time interval $\left[k T_{s},(k+1) T_{s}\right)$ to the train dynamics (2). Update the train's current state including velocity, in-train dynamics and position at the track. Update current time $k=k+1$, and go to step 2).

\section{Simulation}

The train's configurations are the same as in [13]. The rail track information from Spoornet, a South African train operation company, is used (see Fig. 1, in which abscissa is train's relative position on the track). The length of the track is $17 \mathrm{~km}$. There are four locomotives in the train, two of which are located at front, and the other two at the rear of the train. Between those locomotives, 200 wagons are connected oneby-one in the middle. The maximum/minimum absolute value of in-train forces is set to $\pm 2000 \mathrm{kN}$, with regard to the train's operation safety. Simulations are run to investigate impacts of the penalty factors $K_{b}$ and $K_{d}$ and impacts of dynamic $K_{v}$ in the scheduling. The train performance indicators, as described in the cost function, are in-train forces, velocity tracking, coupler fatigue, and energy consumption. The variable $c$ in (9) is set to 2 for investigating the impacts of dynamic $K_{v}$ method. $T_{s}=20 s$ is used as sampling period for the controller. The optimization and control horizons, $N_{p}$ and $N_{c}$, chosen here are for demonstration purpose only as these values can be selected by following standard MPC controller tuning procedures. More detailed comparison of train performance change with regard to $N_{p}$ and $N_{c}$ settings can be found in [24].

The MPC controller is implemented as described in Section III, where the QP problem formulated is solved by following Hildreth's quadratic programming procedure [35]. The newly developed fast convex optimization approach [36] was also tested. In this study, due to the fact that the optimization horizon and control horizon are chosen to be short, the computation time from the Hildreth's approach and the one presented in [36] does not differ much. Computation time required to solve the problem by Hildreth's procedure is given in the following context.

Simulation results with different configurations are summarized in Table I. Regarding the weights in the cost function as well as optimization horizons, $K_{v}=60, K_{e}=10, K_{f}=10$, $N_{p}=4$, and $N_{c}=2$ are fixed in simulations owing to the fact that the focus here is to investigate impacts of the penalty factors and dynamic $K_{v}$ method. In Table I, $|\delta \bar{v}|$ is the absolute value of the difference between the reference velocity and the mean value of all the cars' velocities, $\left|\bar{f}_{i n}\right|$ is the mean value of the absolute values of all the couplers' in-train forces, $E$ is the energy consumption calculated by $E=\int_{0}^{T}|u v| d t$, and 


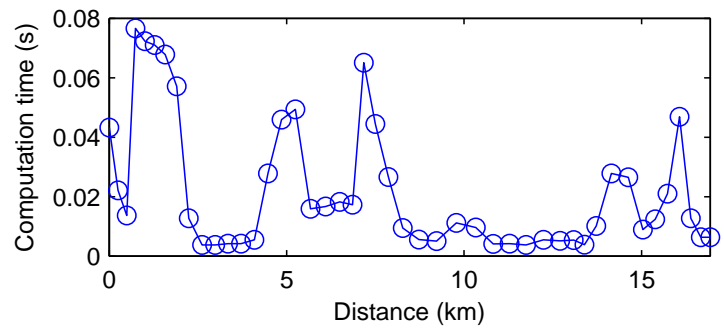

Fig. 2: Computation time

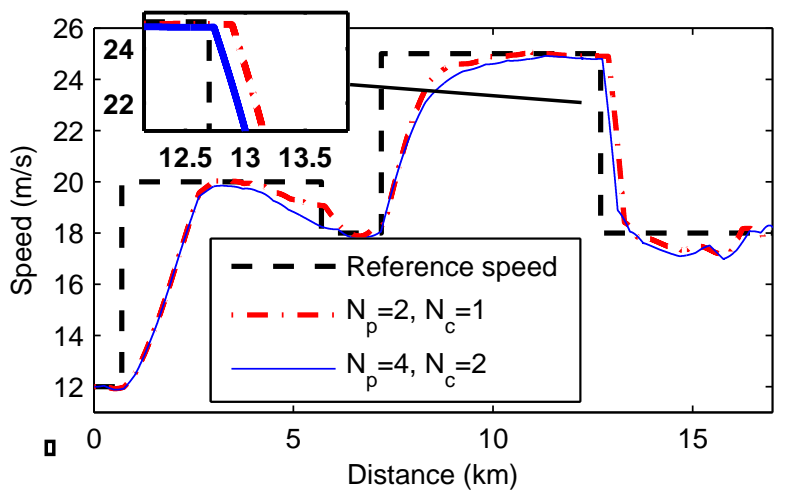

Fig. 3: Performance comparison with different $N_{p}$ and $N_{c}$ while $K_{b}=10$

$\sum \bar{x}_{i n}^{2}$ is the summation of the mean rate of change of all couplers' length in the train over the whole travel.

First of all, to verify that the MPC approach designed with cascade mass point train model is practically applicable, the following simulations are conducted. The computation time for solving the optimization problem at each sampling instant during the simulation with configuration $K_{v}=60, K_{e}=10$, $K_{f}=10, N_{p}=4, N_{c}=2, K_{d}=0$ and $K_{b}=10$ is shown in Fig. 2, which explicitly shows that the computation time is much less than one second. In comparison to the sampling period $T_{s}=20 \mathrm{~s}$, the computation time is very short which means that the MPC approach is practically applicable regarding its time complexity.

\section{A. Impacts of $K_{b}$}

From Table I, it can be seen that a $K_{b}$ larger than one leads to great improvement of the train's performance. Simulation is done when the optimization and control horizons are set to $N_{p}=4$ and $N_{c}=2$. Compared to the results when $K_{b}=1$, both the train's energy consumption and maximum absolute intrain force decrease a lot with a negligible sacrifice of velocity tracking ability when $K_{b}$ is increased to 10 . Specifically, $17 \%$ energy saving (from $25278.58 \mathrm{MJ}$ to $20952.39 \mathrm{MJ}$ ) and $43.5 \%$ decrease of the maximum in-train forces(from $1122.7 \mathrm{kN}$ to $638.08 \mathrm{kN}$ ) are achieved while no considerable change of the velocity tracking indicator is observed.

The above impacts of $K_{b}$ can be explained as the presence of $K_{b}$ punishes wagons' braking forces in a train, which leads to two most noticeable performance changes

1) By punishing the braking forces of wagons, energy dissipated during braking is reduced. For instance, when
$K_{b}=10$ is applied, energy dissipated by wagons' braking is reduced from $15877.28 \mathrm{MJ}\left(K_{b}=1\right)$ to 13684.21MJ. A reduction of $13.8 \%$ is reached.

2) The maximum absolute value of in-train forces and the rate of coupler length change reduce, and as a result, braking occurs less frequently with a large $K_{b}$.

However, it is not always desirable to reduce brake forces. Reasonable brake forces must be applied to a train when necessary. Otherwise, the train's speed tracking performance will deteriorate. For instance, sufficient braking is required when the train runs down a hill. The MPC approach, however, can schedule the train's brake/traction power, to slow down the train before where large braking forces are required to do so taking advantage of its predicted control. If the optimization horizon is long enough, the energy consumed by braking can be reduced as much as possible by the MPC controller.

This feature of MPC gives a way to reduce braking efforts of the train and makes it possible to compensate the speed tracking deterioration resulting from braking penalization, by means of optimizing the train's operation in a long run. As shown in Fig. 3, if the windows are increased from $N_{p}=2$ and $N_{c}=1$ to $N_{p}=4$ and $N_{c}=2$, the train's speed tracking deterioration caused by applying $K_{b}$, especially when the train is required to slow down, reduces significantly. In the case shown in Fig. 3, the train applies more effective braking to bring the train down to the set speed at position $12 \mathrm{~km}$, much faster with a larger $N_{p}$.

\section{B. Impacts of $K_{d}$}

Comparing the results between the first row $\left(K_{d}=0\right)$ and the third row $\left(K_{d}=10\right)$ in Table I, it can be seen that, while velocity tracking results are almost the same, other two indicators change remarkably. On the one hand, energy consumption decreases when a nonzero $K_{d}$ is presented. On the other hand, the maximum absolute in-train forces, increase significantly when $K_{d}=10$ compared to that when $K_{d}=0$. Comparing the second and the third row of Table I, it is manifest that the energy consumption reduction resulted from punishing coupler damping is less than that resulted from reducing braking forces. The coupler cyclic vibration indicator reduces from $0.199 \mathrm{~m}^{2} / \mathrm{s}^{2}$ to $0.177 \mathrm{~m}^{2} / \mathrm{s}^{2}$ when $K_{d}$ increases from 0 to 10 .

Primarily, $K_{d}$ prevents the cyclic length change of couplers in the train in order to alleviate coupler fatigue and reduce the chance of coupler damage. As such, a nonzero $K_{d}$ results in slow length change of couplers, which implies that energy dissipated during coupler damping is reduced and coupler fatigue is alleviated. By increasing $K_{d}=10$, comparing to the base case where $K_{d}=0$ and $K_{b}=1$, an $11.1 \%$ reduction in the rate of coupler length change is achieved. More data supporting this result are shown in Table II. A nonzero $K_{d}$, in contrast, also leads to the result that in-train forces get larger compared to when a zero $K_{d}$ is adopted. This is because release of in-train forces by coupler damping becomes more difficult when a nonzero $K_{d}$ is used. 
TABLE I: Performance comparison while $K_{v}=60, K_{e}=10, K_{f}=10$, and $f=10$

\begin{tabular}{|c|c|c|c|c|c|c|c|c|c|c|c|}
\hline & \multirow[b]{2}{*}{$K_{d}$} & \multirow[b]{2}{*}{$K_{b}$} & \multicolumn{3}{|c|}{$|\delta \bar{v}|(\mathrm{m} / \mathrm{s})$} & \multicolumn{3}{|c|}{$\left|\bar{f}_{\text {in }}\right|(\mathrm{kN})$} & \multicolumn{2}{|c|}{$E(\mathrm{MJ})$} & \multirow{2}{*}{$\sum \dot{x}_{i n}^{2}\left(\mathrm{~m}^{2} / \mathrm{s}^{2}\right)$} \\
\hline & & & $\max$ & mean & std & $\max$ & mean & std & wagon & total & \\
\hline \multirow{4}{*}{ no dynamic $K_{v}$} & 0 & 1 & 8.06 & 1.79 & 2.17 & 1122.70 & 275.03 & 76.46 & 15877.28 & 25278.58 & 0.199 \\
\hline & 0 & 10 & 8.05 & 1.44 & 2.21 & 634.08 & 147.37 & 36.67 & 13684.21 & 20952.39 & 0.158 \\
\hline & 10 & 1 & 8.04 & 1.70 & 2.11 & 1279.44 & 255.94 & 82.83 & - & 21795.41 & 0.177 \\
\hline & 10 & 10 & 7.97 & 1.52 & 2.19 & 1039.82 & 207.08 & 67.04 & - & 21326.38 & 0.138 \\
\hline dynamic $K_{v}$ & 10 & 10 & 8.76 & 1.82 & 2.33 & 810.43 & 269.29 & 56.63 & 12943.80 & 19767.77 & 0.131 \\
\hline
\end{tabular}

TABLE II: $K_{d}$ 's impact on coupler fatigue indicator

\begin{tabular}{l|c|c|c|c}
\hline \hline$K_{d}$ & 0 & 10 & 40 & 60 \\
\hline$\sum \overline{\dot{x}}_{i n}^{2}$ & 0.1988 & 0.1773 & 0.1628 & 0.1549 \\
\hline \hline
\end{tabular}

\section{Combination of $K_{b}$ and $K_{d}$}

As analyzed in the section above, it can be seen from Table I that

1) the maximum absolute in-train force becomes larger when a nonzero $K_{d}$ is used;

2) the maximum absolute in-train forces gets smaller when a larger $K_{b}$ is introduced; and

3) introduction of both $K_{b}$ and $K_{d}$ leads to reduction in energy consumption.

Therefore the potential for improving train performance by combining $K_{b}$ and $K_{d}$ is investigated, results of which are given in the fourth row of Table I. It can be concluded according to the first four rows in this table that the performance indicators on velocity tracking, in-train forces, and energy consumption are the best when only $K_{b}$ is presented. However, according to the coupler fatigue indicator, the rate of change of the coupler length sums up to $0.158 \mathrm{~m}^{2} / \mathrm{s}^{2}$ when $K_{d}=0$, which is higher than $0.138 \mathrm{~m}^{2} / \mathrm{s}^{2}$ when $K_{d}=10$. Thus a compromise leads to the most acceptable result, with both $K_{b}$ and $K_{d}$ employed. In summary, combining the braking and damping penalization can yield better train performance than solo use of each of them. It is up to the train operator's decision whether a larger $K_{d}$ is to be used or the results with $K_{b}$ only are the best. A trade-off must be done.

\section{Impacts of dynamic $K_{v}$}

The idea of dynamically varying $K_{v}$ is to change the weight of velocity tracking according to the train's present velocity tracking state. If the train's velocity tracking indicator is good enough, then the weight on this indicator should be reduced accordingly, so that the other indicators of the train performance can be improved. In addition, the optimization tends to schedule the train to stop in some circumstances when $K_{v}$ is always large owing to the fact that the velocity tracking related term in the cost function can be minimized by minimizing $v_{i}, i=1, \ldots, n$. This is the motivation of applying the dynamic $K_{v}$.

The last two rows in Table I gives a comparison between the results with and without dynamic $K_{v}$. It is evident that with a dynamically varying $K_{v}$, the train's velocity tracking result deteriorates a little while the other two performance indicators get much better. The maximum absolute in-train
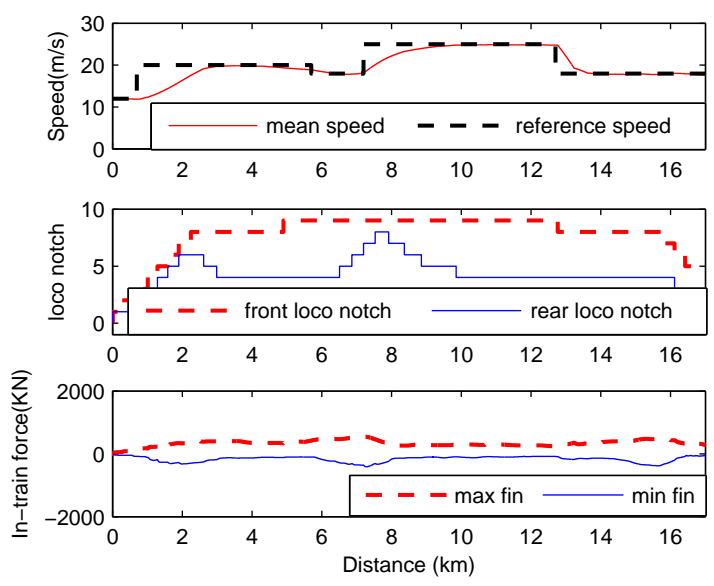

Fig. 4: Results with dynamic $K_{v}$ and $K_{d}=K_{b}=20$

force gets smaller (a reduction of $22.0 \%$ was seen) and energy consumption gets less (reduced by $7.3 \%$ ).

As summary of this section, simulations verified that the train's performance can be improved as expected by only presenting a braking penalty or only presenting a damping penalty. However, combining those two penalties can yield better results. A trade-off must be done when selecting the values of $K_{b}$ and $K_{d}$. In addition, the train's performance can be further improved by a dynamic $K_{v}$. Fig. 4 shows the results when dynamic $K_{v}$ is applied together with both $K_{d}$ and $K_{b}$ set to 20 (the optimization and control horizons are set to $N_{p}=6$ and $N_{c}=4$ ). It is clear that the performance of the train is better in this case than that shown in Fig. 3.

\section{E. Performance on a long uphill track}

The results presented in the simulations above could be unconvincing as it is intuitive that more brake effort is required for a train when it is running on a downhill track than on a uphill track. Therefore, the energy saving and other performance improvement on an uphill track, which is adopted from [15], is investigated in this subsection. The track has a fluctuated upward trend in this case and is much longer than the downhill one used earlier in this section.

The reference speed profile is also adopted from [15]. The train's speed tracking result by the approach presented in this paper and the track information are depicted in Fig. 5. It is seen that the train does not track the speed profile designed in [15] with zero error. The reason is that the speed profile was designed according a single mass point train model where no in-train dynamics are considered while the train operation 

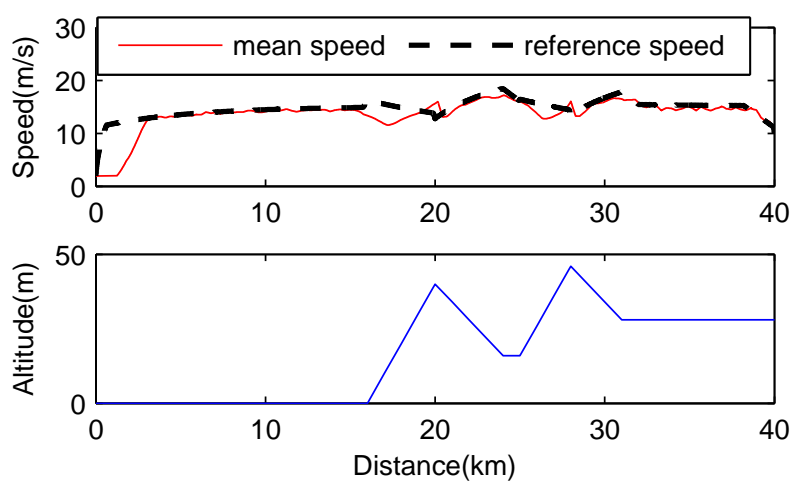

Fig. 5: Performance on a upward track

controller presented in this study schedules the train with intrain forces accounted for. It is clear from Fig. 5 that the biggest deviation from the set speed is observed when the train is running over a hill. This also verifies that the train operation strategy, based on the single mass point model, can lead to unreasonable results. It also confirms that in-train dynamics, such as in-train forces, are of great concern, especially when the train is running over the top of a hill. The speed deviation at the beginning of the schedule shown in Fig. 5 is due to the limit on rate of change of the train's locomotive power.

To demonstrate the advantages of the method presented in this study, the train's performance indicators with and without braking penalty, coupler damping penalty, and the dynamic weighting for speed tracking are compared on this uphill section. It is shown by simulations that the approach proposed yields better results in terms of the mean speed tracking error, in-train force, energy consumption and coupler fatigue indicators, when compared to our previous work [24] where no penalties and dynamic weighting methods are employed. In particular, the control proposed in this study reduces $\left|\bar{f}_{i n}\right|$ by $21.85 \%$ from $1353.57 \mathrm{kN}$ to $1057.84 \mathrm{kN}$; the energy consumption $E$ is also reduced remarkably by $24.87 \%$ from 72014.79 MJ to $54104.54 \mathrm{MJ}$; the coupler fatigue indicator, $\sum \bar{x}_{i n}^{2}$, decreases from 0.163 to 0.133 (18.40\% reduction) as well. Meanwhile, mean velocity tracking error increased a little by $0.64 \%$ from $11.21 \mathrm{~m} / \mathrm{s}$ to $11.28 \mathrm{~m} / \mathrm{s}$.

The results shown in this subsection verify that the the proposed approach is able to achieve better train performance with regard to the performance indicators considered, regardless of the rail track condition.

\section{DISCUSSION}

In this section, the proposed penalties and dynamic $K_{v}$ method are further analyzed, together with some suggestions on their applications.

As for the braking penalization, it is always recommended to add a larger $K_{b}$ if the train is running on a relatively flat track or on a track with a slight downward gradient, in order to reduce energy consumption and reduce the maintenance/replacement cost of braking linings. A moderate value of $K_{b}$ should be used if a train is running on a fluctuating track, because the train can make use of the track profile to slow itself down under such circumstances. On the contrary, if the train is running down a steep continuous downward track, one should use a small value of $K_{b}$ so that the train can operate safely.

Regarding the penalty factor for coupler damping, the choice of $K_{d}$ is indirectly related to the damping coefficients, $d$, of couplers in use (refer to [21] for details). A larger $d$ means the coupler can dissipate more energy by damping. Therefore, adding $K_{d} \dot{x}_{i n_{i}}^{2}$ in the cost function offsets the effect of $d$. This suggests that when the damping effects of couplers mounted on a train are large, a large $K_{d}$ can be used without jeopardizing the train's other performance, while reducing its energy consumption and relieving coupler fatigue. Otherwise, if damping effects of couplers are weak (i.e. $d$ is small), a small $K_{d}$ should be used to prioritize the train's operation safety (maintain in-train forces within the safe range).

When it comes to dynamic $K_{v}$, the maximum value of $K_{v}^{\prime}=K_{v}$ can be chosen as done by [22] to consider the trade-offs between different aspects of the train performance. It is the rate of change of $K_{v}^{\prime}$ that is the concern here. This relies on the specified tolerable speed tracking error. For instance, if one sets the maximum tolerable speed tracking error $\left|v-v_{r}\right|$ to $M$, which means that $K_{v}^{\prime}=K_{v}$ apply when $\left|v-v_{r}\right| \geq M$, then $K_{v}$ can vary according to $\left|v-v_{r}\right|$ when $\left|v-v_{r}\right|<M$. Firstly, it is clear from (9) that if $\left|v-v_{r}\right| \rightarrow 0$, then $K_{v}^{\prime} \rightarrow 0$ regardless of the value of $c$. Secondly, according to the settings, if $\left|v-v_{r}\right| \geq M$ then $K_{v}^{\prime}=K_{v}$. In view of the above two points, one gets

$$
\lim _{\left|v-v_{r}\right| \rightarrow M} K_{v}\left(1-e^{-\frac{\left(v-v_{r}\right)^{2}}{c^{2}}}\right)=K_{v} .
$$

This further implies that $e^{-\frac{M^{2}}{c^{2}}}=0$, which is not possible. If this equation is modified, from an engineering perspective, to be $e^{-\frac{M^{2}}{c^{2}}}<10^{-3}$, one can immediately get $c^{2}<\frac{M^{2}}{6.9}$. Therefore, if one chooses $M=6$, then $c^{2}$ should be less than 5.22. This provides a way to find a proper value of $c^{2}$. In such a way, one can choose $M$ first and then determine a suitable $c$. The presence of $c$, together with $K_{v}$, gives flexibility for users to specify the train's speed tracking performance. However, the key contribution of dynamic $K_{v}$ is that the velocity tracking term in the cost function becomes nearly zero when speed tracking is good enough. This offers more opportunities for improvement of the train's overall performance.

\section{CONCLUSION}

An MPC approach for heavy haul trains' operation is presented. Two penalty factors are introduced to improve the trains performance in terms of energy consumption, speed tracking, operation safety, and coupler fatigue. One penalty factor is introduced for the braking forces of wagons in the train and the other for the couplers' damping effects. In addition, a dynamic weighting method is proposed for the weight of the train's velocity tracking indicator term in the cost function of the problem, to improve the train's overall performance.

As is verified by simulations, the penalty factors and the dynamically varying $K_{v}$ method presented can improve the 
train's performance in terms of velocity tracking, coupler fatigue alleviation, maximum in-train forces reduction and energy consumption minimization. It also shows that the train's performance can be further improved if the penalty factors and the dynamic $K_{v}$ are employed together.

\section{REFERENCES}

[1] M. Matsumoto, "The revolution of train control system in Japan," in 2005 International Symposium on Autonomous Decentralized Systems, Chengdu, China, 2005, pp. 599-606.

[2] B. Nelldal, "Efficient train systems for freight transport-a systems study," KTH Railway Group at Royal Institute of Technology, Stockholm, Report 0505, 2005.

[3] S. Mu and M. Dessouky, "Scheduling freight trains traveling on complex networks," Transportation Research Part B: Methodological, vol. 45, no. 7, pp. 1103 - 1123, 2011.

[4] Y. Fu, Z. Gao, and K. Li, "Optimization method of energy saving train operation for railway network," Journal of Transportation Systems Engineering and Information Technology, vol. 9, no. 4, pp. $90-96$ 2009.

[5] M. Dorfman and J. Medanic, "Scheduling trains on a railway network using a discrete event model of railway traffic," Transportation Research Part B: Methodological, vol. 38, no. 1, pp. 81 - 98, 2004.

[6] X. Zhuan and X. Xia, "Fault-tolerant control of heavy-haul trains," Vehicle System Dynamics, vol. 48, no. 6, pp. 705-735, 2010.

[7] P. Howlett, P. Pudney, and X. Vu, "Local energy minimization in optimal train control," Automatica, vol. 45, no. 11, pp. 2692-2698, 2009.

[8] X. Yang, X. Li, Z. Gao, H. Wang, and T. Tang, "A cooperative scheduling model for timetable optimization in subway systems," IEEE Transactions on Intelligent Transportation Systems, vol. 14, no. 1, pp. 438-447, 2013.

[9] X. Zhuan and X. Xia, "Optimal scheduling and control of heavy haul trains equipped with electronically controlled pneumatic braking systems," IEEE Transactions on Control Systems Technology, vol. 15, no. 6, pp. 1159-1166, 2007.

[10] R. Liu and I. M. Golovitcher, "Energy-efficient operation of rail vehicles," Transportation Research Part A: Policy and Practice, vol. 37, no. 10, pp. 917-932, 2003.

[11] P. Gruber and M. Bayoumi, "Suboptimal control strategies for multilocomotive powered trains," IEEE Transactions on Automatic Control, vol. 27, no. 3, pp. 536-546, 1982.

[12] A. Astolfi and L. Menini, "Input/output decoupling problems for high speed trains," in Proceedings of the 2002 American Control Conference, vol. 1, Alaska, USA, 2002, pp. 549-554.

[13] X. Zhuan and X. Xia, "Cruise control scheduling of heavy haul trains," IEEE Transactions on Control Systems Technology, vol. 14, no. 4, pp. 757-766, Jul. 2006.

[14] P. Howlett, "Optimal strategies for the control of a train," Automatica, vol. 32, no. 4, pp. 519-532, 1996.

[15] E. Khmelnitsky, "On an optimal control problem of train operation," IEEE Transactions on Automatic Control, vol. 45, no. 7, pp. 1257-1266, 2000.

[16] R. Chevrier, "An evolutionary multi-objective approach for speed tuning optimization with energy saving in railway management," in 13th International IEEE Conference on Intelligent Transportation Systems, Madeira Island, Portugal, 2010, pp. 279 -284.

[17] M. Miyatake and H. Ko, "Optimization of train speed profile for minimum energy consumption," IEEJ Transactions on Electrical and Electronic Engineering, vol. 5, no. 3, pp. 263-269, May 2010.

[18] X. Li, Z. Gao, and W. Sun, "Existence of an optimal strategy for stochastic train energy-efficient operation problem," Soft Computing, vol. 17 , no. 4, pp. 651-657, 2013.

[19] X. Li, D. Wang, K. Li, and Z. Gao, "A green train scheduling model and fuzzy multi-objective optimization algorithm," Applied Mathematical Modelling, vol. 37, no. 4, pp. 2063 - 2073, 2013.

[20] X. Zhuan, "Optimal handling and fault-tolerant speed regulation of heavy haul trains," Ph.D Thesis, University of Pretoria, Pretoria, 2007.

[21] M. Chou, X. Xia, and C. Kayser, "Modelling and model validation of heavy-haul trains equipped with electronically controlled pneumatic brake systems," Control Engineering Practice, vol. 15, no. 4, pp. 501509, 2007.

[22] X. Zhuan and X. Xia, "Speed regulation with measured output feedback in the control of heavy haul trains," Automatica, vol. 44, no. 1, pp. 242 $247,2008$.
[23] L. Zhang, X. Zhuan, and X. Xia, "Optimal operation of heavy haul trains using model predictive control methodology," in 2011 IEEE International Conference on Service Operations, Logistics, and Informatics, Beijing, China, 2011, pp. $402-407$.

[24] L. Zhang and X. Zhuan, "Optimal operation of heavy haul trains equipped with ECP brake systems using model predictive control methodology," IEEE Transactions on Control Systems Technology, vol. 22, no. 1 , pp. $13-22,2013$.

[25] X. Xia, J. Zhang, and A. Elaiw, "An application of model predictive control to the dynamic economic dispatch of power generation," Control Engineering Practice, vol. 19, no. 6, pp. 638 - 648, 2011.

[26] X. Zhuan and X. Xia, "Development of efficient model predictive control strategy for cost-optimal operation of a water pumping station," IEEE Transactions on Control Systems Technology, vol. 21, no. 4, pp. 14491454, 2013.

[27] S. Qin and T. A. Badgwell, "A survey of industrial model predictive control technology," Control Engineering Practice, vol. 11, no. 7, pp. $733-764,2003$

[28] A. Elaiw, X. Xia, and A. Shehata, "Application of model predictive control to optimal dynamic dispatch of generation with emission limitations," Electric Power Systems Research, vol. 84, no. 1, pp. 31 - 44, 2012.

[29] Y. Bai, B. Mao, F. Zhou, Y. Ding, and C. Dong, "Energy-efficient driving strategy for freight trains based on power consumption analysis," Journal of Transportation Systems Engineering and Information Technology, vol. 9, no. 3, pp. 43 - 50, 2009.

[30] S. P. Chunduru, M. J. Kim, and C. Mirman, "Failure analysis of railroad couplers of AAR type E," Engineering Failure Analysis, vol. 18, no. 1, pp. $374-385,2011$.

[31] Y. Wang, B. D. Schutter, T. J. van den Boom, and B. Ning, "Optimal trajectory planning for trains $\mathrm{c}$ a pseudospectral method and a mixed integer linear programming approach," Transportation Research Part C: Emerging Technologies, vol. 29, no. 0, pp. 97 - 114, 2013.

[32] S. Lu, S. Hillmansen, T. K. Ho, and C. Roberts, "Single-train trajectory optimization," IEEE Transactions on Intelligent Transportation Systems, vol. 14 , no. 2 , pp. 743-750, 2013.

[33] L. Zhang and X. Zhuan, "Braking-penalized receding horizon control of heavy-haul trains," IEEE Transactions on Intelligent Transportation Systems, vol. 14, no. 4, pp. 1620-1628, 2013.

[34] L. Zhang, Q. Li, and X. Zhuan, "Energy-efficient operation of heavy haul trains in an mpc framework," in 2013 IEEE International Conference on Intelligent Rail Transportation, Beijing, China, 2013.

[35] D. G. Luenberger, Optimization by Vector Space Methods. New York: John Wiley and Sons, 1969.

[36] J. Mattingley and S. Boyd, "Real-time convex optimization in signal processing," Signal Processing Magazine, IEEE, vol. 27, no. 3, pp. 50$61,2010$.

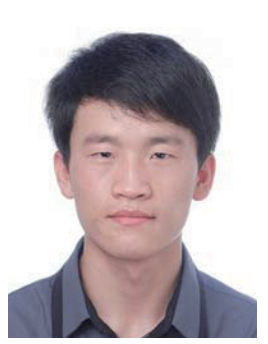

Lijun Zhang received his B.Eng and M.Eng from School of Power and Mechanical Engineering, Wuhan University, China, in 2010 and 2012, respectively.

He is currently a Lecturer and Ph.D candidate at the University of Pretoria, South Africa. His research interests include energy modeling, optimal operation control and monitoring of power and industrial systems.

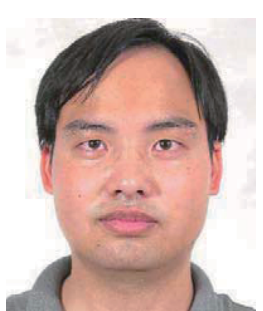

Xiangtao Zhuan obtained his B.Eng from Wuhan University of Hydraulic and Electrical Engineering, China in 1999. He received a master's degree from Wuhan University in 2002. He obtained his $\mathrm{PhD}$ degree at the University of Pretoria in 2007.

$\mathrm{He}$ is currently a Professor and the head of Department of Automation at Wuhan University. His current research interests include modeling and optimal control of power systems, heavy haul trains and water pumping stations. 